\title{
THE INTEGRATED CIRCUITS PHOTOMASKS IMAGES ALIGNEMENT FOR AUTOMATED OPTICAL INSPECTION SYSTEM
}

\author{
ANTOSHCHUK, S.; KRYLOV, V. \& SHCHERBAKOVA, G.
}

Abstract: The automated optical inspection system for integrated circuits photo masks is designed. This low error probability system based on application of hyperbolic wavelet transforms and integrated circuits photo masks fiducial marks geometric moment features. The experimental results proved the high noise stability properties, which allow reducing lighting apparatus and high precision mechanism conditions.

Key words: automated optical inspection system, hyperbolic wavelet transforms, integrated circuits, photo masks, alignment
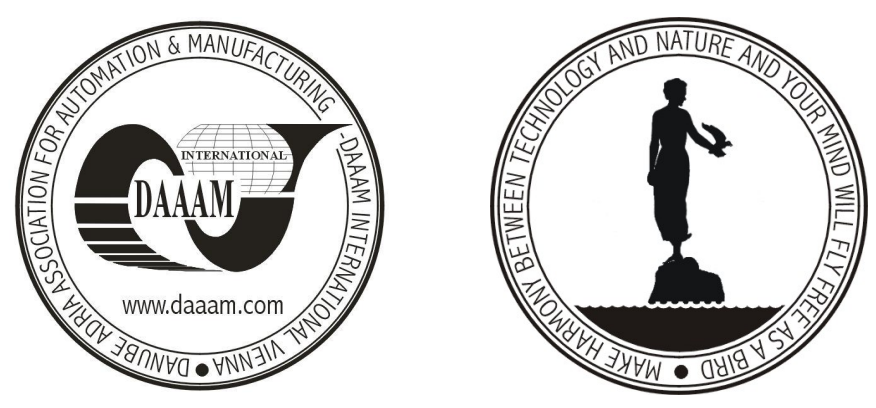

Authors' data: Prof. Antoshchuk, S[vetlana]; Dr. Sc. Krylov, V[ictor]; Master of Science Shcherbakova, G[alina], Odessa National Polytechnic University, Odessa, Ukraine,svetlana_onpu@mail.ru,milit@milit.tm.odessa.ua, is@favt.ospu.odessa.ua

This Publication has to be referred as: Antoshchuk, S.; Krylov, V. \& Shcherbakova, G. (2007). The integrated circuits photomasks images alignement for automated optical inspection system, Chapter 26 in DAAAM International Scientific Book 2007, B. Katalinic (Ed.), Published by DAAAM International, ISBN 3-90150960-7, ISSN 1726-9687, Vienna, Austria

DOI: $10.2507 /$ daaam.scibook.2007.26 